\title{
Isolation and identification of a distinct strain of Culex Flavivirus from mosquitoes collected in Mainland China
}

Wang Huanyu', Wang Haiyan², Fu Shihong ${ }^{1}$, Liu Guifang ${ }^{2}$, Liu Hong ${ }^{1}$, Gao Xiaoyan', Song Lizhi², Simon Rayner ${ }^{3}$, Xu Aiqiang ${ }^{2}$ and Liang Guodong ${ }^{1 *}$

\begin{abstract}
Background: Culexflavivirus (CXFV) is an insect specific virus that has been isolated from Culexpipiens, Culexquinquefasciatus, Culextritaeniorhynchus and other Culex mosquitoes. It is a novel flavivirus isolated in Asia, North America, Central America and Africa. Phylogenetic analysis indicates that, based on the envelope gene ( $E$ gene) sequence, the worldwide CxFV strains can be divided into two genotypes.

Result: A virus (SDDM06-11) was isolated from Culexpipiens collected in Shandong Province, China in 2006. The strain caused cytopathic effect (CPE) in Aedesalbopictus (C6/36) cells by 3 days post-infection and immunofluorescence assay (IFA) showed a reaction with Japanese encephalitis virus (JEV) polyclonal antibodies. Phylogenetic analysis of the E gene sequence showed CXFV formed two genotypes with the SDDM06-11 strain assigned to genotype 1. Analysis of the E gene nucleotide homology showed the virus possessed characteristic amino acids at specific sites. The nucleotide homology of the open reading frame (ORF) was $94.8 \%-95.1 \%$ between SDDM06-11 and isolates from Japan, lowa and Texas, and 90.2\%-90.5\% between SDDM06-11 and isolates from Uganda and Mexico.
\end{abstract}

Conclusion: In this paper we report the first isolation and identification of an isolate of CXFV in mainland China. Phylogenetic analysis indicates the isolate belongs to genotype 1. Our findings provide insight into the occurrence of CxFV in Culex mosquito populations and its distribution on a global scale.

Keywords: CulexFlavivirus, Genotype, Mosquitoes

\section{Background}

The genus Flavivirus in the family Flaviviridae is a group of single stranded positive sense RNA viruses that are primarily arthropod borne [1]. Phylogenetic analysis classifies these viruses into mosquito-borne, tick-borne, no known vector and viruses that are specific to insects, but which do not infect vertebrates [2,3]. This final group includes cell fusing agent virus (CFAV) [4], Kamiti River virus (KRV)[5] and Culexflavivirus (CxFV)[6].

$\mathrm{CxFV}$ is an insect-specific virus that can replicate in C6/36 cells but not in other mammalian cell lines.

\footnotetext{
* Correspondence: gdliang@hotmail.com

'State Key Laboratory for Infectious Disease Prevention and Control, Department of Viral Encephalitis, Institute for Viral Disease Control and Prevention, Chinese Center for Disease Control and Prevention, Beijing 102206, People's Republic of China

Full list of author information is available at the end of the article
}

CxFV was first isolated in 2003 in Japan (in species Culexpipiens and Culextritaeniorhynchus) and then in Indonesia in 2004 (Culexquinquefasciatus)[6]. Subsequently the virus has been isolated in North America: Iowa and Texas in the United States [7,8] (2007-2008, Culexquinquefasciatus, Culexrestuans); Central America: Guatemala (2006, Culexquinquefasciatus) [9], the Yucatan peninsula in Mexico [10,11] (2007-2008, Culexquinquefasciatus, Culexinterrogator); Trinidad in the West Indies [8] (2008, Culexquinquefasciatus); and Uganda, Africa [12] (2008, Culexquinquefasciatus). E gene phylogenetic analysis of these isolates showed that they can be clearly divided into two genotypes $[8,9]$.

Relatively few flaviviruses have been isolated in China, the most common of which are Japanese encephalitis virus (JEV), dengue virus (DENV) and tick-borne

\section{Biomed Central}


encephalitis virus (TBEV) with presence of associated disease symptoms $[13,14]$. In recent years, a novel insect-specific Flavivirus was isolated from Aedesvexans in rural corrals in Chaoyang city in Liaoning province in China [15], but the distribution and significance of these insect specific viruses remains unclear in China.

In this study, we report the isolation and identification of a distinct strain of CxFV from Culexpipiens mosquitoes collected in Shandong Province in China in 2006 and perform nucleotide sequencing analysis in order to compare with other $\mathrm{CxFV}$ isolates previously collected from around the world.

\section{Methods}

\section{Mosquito collection}

Mosquitoes used in this study were collected in August in 2006 in Dongming county, Shandong province, China. Mosquitoes were collected using light traps (Hubei Lucky Star Environment Protection Co., Ltd.) in fields, pig farms and dwellings. The traps were placed before sunset and mosquitoes were collected from the traps the following morning after sunrise. Collected mosquitoes were frozen for $30 \mathrm{~min}$ at $-20^{\circ} \mathrm{C}$ then placed on an ice plate to determine mosquito species and exclude blood-fed and/or male mosquitoes [16]. The mosquitoes were sorted according to species with 50 to 100 adults per pool, transported on dry ice and then stored in liquid nitrogen in the laboratory.

\section{Cell culture and virus isolation}

Mosquito cell line C6/36 and baby hamster kidney (BHK-21) cell line were used for virus isolation. The two cell lines were cultured with Eagle's minimum essential medium (MEM) (Sigma-Aldrich, USA) with $10 \%$ heat-inactivated fetal bovine serum (FBS) (Sigma), $2 \%$ non-essential amino acid (Sigma), $100 \mathrm{U} / \mathrm{ml}$ penicillin (Gibco, Invitrogen, USA), $100 \mu \mathrm{g} / \mathrm{ml}$ streptomycin (Gibco), and maintained at $28^{\circ} \mathrm{C}$ (for mosquito cells) and $37^{\circ} \mathrm{C}$ (for mammalian cells) with $5 \% \mathrm{CO}_{2}$.

Pools of mosquitoes were homogenized using a Mixer Mill (Tissuelyser, Qiagen, Germany) and $3 \mathrm{~mm}$ stainless steel beads in $2 \mathrm{~mL}$ sterile plastic tubes and $1.5 \mathrm{~mL}$ of MEM containing $2 \%$ fetal bovine serum, $2 \mathrm{mM} \mathrm{L}$-glutamine, 50 units $/ \mathrm{mL}$ Penicillin and $100 \mathrm{ug} / \mathrm{mL}$ Streptomycin (Gibco Cat No 15140). Samples were centrifuged at $12,000 \mathrm{rpm}$ for 20 minutes at $4^{\circ} \mathrm{C}$. Then, $200 \mu \mathrm{l}$ of the clarified homogenates were inoculated into single 5.5 $\mathrm{cm}^{2}$ Nunc tubes (Nunclon, Denmark) spread with a monolayer of C6/36 and BHK-21 cells respectively for 1 $\mathrm{h}$ at constant temperature. After discarding and refreshing with $500 \mu \mathrm{l}$ medium, the cell cultures were incubated with the same conditions for 6-7 days. Cytopathic effects (CPE) were checked every 8 hours after incubation for 24 hours and observation over the next 6-7 days. At 70\% CPE (3 days after post-infection), the culture supernatants were harvested and cellular debris was removed by centrifugation at $12,000 \mathrm{rpm}$. The supernatants were stored at $-80^{\circ} \mathrm{C}$ until identification.

\section{Indirect immunofluorescence assay}

Virus isolate in cell culture was identified by indirect immunofluorescence assay (IFA). Infected and uninfected cell suspensions were applied to Teflon-coated 10-well slides, which were then air dried and fixed in acetone. The IFA was conducted with a panel of antiserum with fluorescein isothiocyanate-conjugated goat anti-mouse IgG as the second antibody (Sigma) by previously described procedures [17]. The panel of polyclonal antibody (JEV-GSS) against JEV was prepared by our laboratory [18].

\section{RT-PCR and nucleotide sequencing}

Viral RNA was extracted from $140 \mu$ l of virus culture stocks using the QIAamp viral RNA extraction kit (Qiagen, Valencia, CA, USA) in accordance with the manufacturer's protocol and stored at $-70^{\circ} \mathrm{C}$ until use. Briefly, purified RNA was used as template to finish the reverse transcription-polymerase chain reactions (RT-PCR) for cDNA synthesis using Ready-To-Go ${ }^{\mathrm{TM}}$ You-Prime FirstStrand Beads (Amersham Biosciences, Piscatway, NJ, USA) and supplied random hexanucleotide primers. Flavivirus genus-specific primer sets [3] were used for identification. Then ten overlapping primers (Table 1) were designed from the Tokyo strain genome sequence (GenBank number: AB262759) to amplify the complete open reading frame (ORF) nucleotide sequence of the viral genomic RNA.

To avoid any potential contamination, JEV RNA and water were used as positive and negative controls respectively at all stages. RT-PCR products were purified using a commercial kit (ExoSAP-IT PCR Purification Kit, USB, USA). PCR products were sequenced using a PRIMS Ready Reaction Dyedeoxy Terminator Cycle Sequencing Kit on an ABI Prism 3100 Avant Genetic Analyser (Applied Biosystems, California, USA) using the PCR primer sets listed in Table 1. Dye terminator sequencing was used for both strands with double coverage and the results were inspected for accuracy and quality. After sequencing we got the ATGC software package (GENETYX Corp., Tokyo, Japan) was used to complete the sequence assembly.

Sequence data for the $E$ and ORF gene of strain SDDM06-11 were deposited in GenBank with accession number JF938690 and JQ518484, respectively.

\section{Sequence analysis and phylogenetic comparisons}

Additional CxFV sequences were downloaded from GenBank, a list of the $49 \mathrm{CxFV}$ isolates used in the 
Table 1 Degenerate primer sequences used for viral screening and primer sets used for SDDM06-11

\begin{tabular}{|c|c|c|c|}
\hline Primer & Position $^{a}$ & Sequence, $5^{\prime}-3^{\prime}$ & Polarity \\
\hline CXFV-SD-1-F & 9 & TGGTTA & Sense \\
\hline CXFV-SD-1-R & $1198-1217$ & GATTGTAGGGCTGGGTTGAG & Reverse \\
\hline CxFV-SD-2-F & 1034-1049 & AACGGACTTCTTGAGTTTCGC & Sense \\
\hline CxFV-SD-2-R & $2197-2217$ & GCCTTGGTGTAGACAAAGTATC & Reverse \\
\hline CXF & $2002-2020$ & GCAAGGT & Sen \\
\hline$-R$ & 56 & GAC & rse \\
\hline D-4-F & 55 & TTCC & Sense \\
\hline CxFV-SD-4-R & $4233-4255$ & CGGTCCGTAAGTTCCTTCTAAT & Reverse \\
\hline CXFV-SD-5-F & 3880-3989 & CTATGCTGTGACGCATCCTG & Sense \\
\hline CXFV-SD-5-R & $6232-6254$ & AAGCCACAAA & Reverse \\
\hline $\mathrm{CxFV}$ & 0040 & ACTCAGTGCTGTTTCAAGG & Sense \\
\hline CxFV-SD-6-R & $7204-7226$ & CTCGTCCTGTTATCTCATCGTC & Reverse \\
\hline CXFV-SD-7-F & 7021-7039 & GATTTCCTGGGAGACGAT & Sense \\
\hline CXFV-SD-7-R & 8319-8337 & TCTCGCAACCTCTTCACG & Reverse \\
\hline CXFV- & 33 & TTC & e \\
\hline R & 44 & G & Reverse \\
\hline CxFV & $9140-9159$ & CTGGACC & Sense \\
\hline CXFV-SD-9-R & $10332-10350$ & TGGCTGCGAGGGTGACTT & Reverse \\
\hline CXFV- & 038 & GGTGAGCGGGACA & Sense \\
\hline CXFV-SD-10-R & 10794-10815 & CCCGCAACAAGTCTCCTAACG & Reverse \\
\hline
\end{tabular}

analyses with origin and year of isolation is shown in Table 2. Nucleotide and amino acid sequence alignments were generated by CLUSTAL_X version 1.8 [19]. The optimal nucleotide substitution model was estimated as the General Time Reversible with rate heterogeneity among sites and invariable sites using Akaike Information Criterion as implemented in jModeltest v.0.1.1 (http://darwin.uvigo.es/software/jmodeltest.html). The phylogenetic tree was computed with the GTR + I $+G$ model using the maximum likelihood approach with the PHYML software package [20]. The statistical significance of the phylogeny was estimated by the nonparametric bootstrap analysis with 100 replicates.

Neighbor Joining (NJ) and Maximum Likelihood (ML) trees were also estimated for the nucleotide alignments using PHYLIP, v3.6-alpha (http://atgc.lirmm.fr/phyml) and MEGA v5.1 with empirical base frequencies, and a gamma distribution to estimate rate variation among sites. Bootstrap values were determined for 100 replicates.

The trees were rooted using a CRFV (GenBank accession no. NC001564) sequence as the outgroup. Analysis of nucleotide and translated amino acid sequence identities for the $\mathrm{E}$ and ORF sequences was performed using the GeneDoc and Lasergene software packages (DNASTAR Inc, USA).

\section{Results}

Virus isolation and identification

A total of 80 pools consisting of 4118 Culextritaeniorhynchus, 839 Culexpipiens and 27 Anopheles sinensis were collected from Dongming county, Shandong province, China, from August 9 to 11, 2006 and were processed for virus isolation. A virus isolate was identified that caused CPE in C6/36 cells and which was characterized by marked syncytia, aggregation and fusion by day 3 post-infection (Figure $1 \mathrm{~A}$ and $1 \mathrm{~B}$ ); no CPE was observed in BHK-21 cells. Antigen IFA using JEV polyclonal antibody (JEV-GSS) identified the isolate as a flavivirus (Figure $2 \mathrm{~A}$ and $2 \mathrm{~B}$ ). RT-PCR amplification using flavivirus primers FU1 and cFD2 [3]. BLASTN analysis against the nr database at NCBI showed the highest scoring hits were all from CxFV with 95\% similarity and an E-value of 0. The isolate was designated SDDM06-11.

\section{Analysis of the $\mathrm{E}$ gene sequence}

Table 3 shows a summary of the nucleotide and amino acid identities for the $\mathrm{E}$ gene according to genotype and geographical location amongst strains used in the phylogenetic analysis.

Specifically, the nucleotide identity between SDDM0611 and genotype 1 strains (Japan, Indonesia and North America) (Iowa and Texas) was 94.4\%-94.8\%, and the corresponding identity of SDDM06-11 with genotype 2 (Uganda, Trinidad, Guatemala and Mexico) was 89.4\%$90.2 \%$. The identity between genotype 1 and genotype 2 was $89.1 \%-90.9 \%$. The within genotype identity for genotype 1 was $94.4 \%-99.6 \%$ and within genotype 2 was 97.9\%-99.2\%. The E gene amino acid identity amongst all the CxFV strains was $97.2 \%-100 \%$ (Table 3).

Examination of the amino acid sequence for the $\mathrm{E}$ gene indicated that each genotype contained site-specific mutations (Table 4). A total of ten mutations were identified that were specific to genotype I and 2; these occurred at E-60 (Val), E-64 (Phe), E-117 (Gly), E-185 (His), E-236 (Val), E-326 (Arg), E-340 (Ile), E381 (His) E-407E (Leu), E-423 (Ile). Mutations that were unique to SDDM06-11 (i.e., that were not present in genotype 1 or 2) occurred at E-125 (Val) and E-414 (Val).

\section{Analysis of the ORF gene sequence}

The nucleotide and deduced amino acid sequences of the ORF of SDDM06-11 were compared with those of six other CxFV strains selected from the GenBank database. The nucleotide identity between SDDM06-11 and other genotype 1 sequences (Japan, Iowa and Texas) was 94.8\%-95.1\%, and between SDDM06-11 and genotype 2 sequences (Uganda and Mexico) was 90.2\%$90.5 \%$. The calculated amino acid identity for all the CxFV strains was 96.5\%-98.2\% (Table 5). The highest 
Table 2 Details of Culexflavivirus (CxFV) strains used in this study

\begin{tabular}{|c|c|c|c|c|c|c|}
\hline \multirow[t]{2}{*}{ No. } & \multirow[t]{2}{*}{ Strain } & \multirow[t]{2}{*}{ Year } & \multicolumn{2}{|c|}{ Geographic Location } & \multirow[b]{2}{*}{ Source/Host } & \multirow[t]{2}{*}{ GenBank accession no. } \\
\hline & & & Country & Location & & \\
\hline 1 & SDDM06-11* & 2006 & China & Dongming & Culexpipiens & JF938690 \\
\hline 2 & Tokyo & 2003 & Japan & Tokyo-Shinjuku & Culexpipiens & NC_008604/AB262759 \\
\hline 3 & NIID-21-2 & 2004 & Japan & Tokyo-Shinjuku & Culexpipiens & AB377213 \\
\hline 4 & Narita & 2003 & Japan & Chiba-Narita & Culexpipiens & AB262760 \\
\hline 5 & Toyama & 2003 & Japan & Toyama & Culexpipiens & AB262761 \\
\hline 6 & Hokkaido & 2003 & Japan & Hokkaido-Abashiri & Culexpipiens & AB262762 \\
\hline 7 & Osaka & 2003 & Japan & Osaka & Culexpipiens & AB262763 \\
\hline 8 & Mie-Cp & 2004 & Japan & Mie-Tsu & Culexpipiens & AB262764 \\
\hline 9 & Morioka & 2003 & Japan & Iwate-Morioka & Culexpipiens & AB262765 \\
\hline 10 & Mie-Ct & 2004 & Japan & Mie-Tsu & Culextritaeniorhynchus & AB262767 \\
\hline 11 & Surabaya & 2004 & Indonesia & Surabaya & Culexquinquefasciatus & AB262766 \\
\hline 12 & Izabal & 2006 & Guatemala & Puerto Rico Izabal & Culexquinquefasciatus & EU805805 \\
\hline 13 & HOU24518 & 2008 & USA & Texas-Harris county & Culexquinquefasciatus & FJ502995 \\
\hline 14 & HOU24519 & 2008 & USA & Texas-Harris county & Culexrestuans & FJ502996 \\
\hline 15 & HOU24284 & 2008 & USA & Texas-Harris county & Culexrestuans & FJ502997 \\
\hline 16 & HOU24471 & 2008 & USA & Texas-Harris county & Culexrestuans & FJ502998 \\
\hline 17 & HOU24516 & 2008 & USA & Texas-Harris county & Culexquinquefasciatus & FJ502999 \\
\hline 18 & HOU24559 & 2008 & USA & Texas-Harris county & Culexquinquefasciatus & FJ503001 \\
\hline 19 & TR3115 & 2008 & West Indies, Trinidad & Champs Fleur & Culexquinquefasciatus & FJ503002 \\
\hline 20 & HOU24522 & 2008 & USA & Texas-Harris county & Culexquinquefasciatus & FJ503000 \\
\hline 21 & TR3116 & 2008 & West Indies, Trinidad & Champs Fleur & Culexquinquefasciatus & FJ503003 \\
\hline 22 & CXFV-Mex07 & 2007 & Mexico & Yucatan State & Culexquinquefasciatus & EU879060 \\
\hline 23 & 1064 & 2007 & USA & lowa & Culexpipiens & FJ663026 \\
\hline 24 & 380 & 2007 & USA & lowa & Culexpipiens & FJ663027 \\
\hline 25 & 383 & 2007 & USA & lowa & Culexpipiens & FJ663028 \\
\hline 26 & 635 & 2007 & USA & lowa & Culexpipiens & FJ663029 \\
\hline 27 & 318 & 2007 & USA & lowa & Culexpipiens & FJ663030 \\
\hline 28 & 377 & 2007 & USA & lowa & Culexpipiens & FJ663031 \\
\hline 29 & 599 & 2007 & USA & lowa & Culexpipiens & FJ663032 \\
\hline 30 & 657 & 2007 & USA & lowa & Culexpipiens & FJ663033 \\
\hline 31 & lowa07 & 2007 & USA & lowa & Culexpipiens & FJ663034 \\
\hline 32 & Uganda08 & 2008 & Uganda & Entebbe & Culexquinquefasciatus & GQ165808 \\
\hline 33 & T955 & 2008 & Mexico & Yucatan Peninsula & Culexinterrogator & GU289683 \\
\hline 34 & M2168 & 2008 & Mexico & Yucatan Peninsula & Culexquinquefasciatus & GU289684 \\
\hline 35 & M2313 & 2008 & Mexico & Yucatan Peninsula & Culexquinquefasciatus & GU289685 \\
\hline 36 & M2605 & 2008 & Mexico & Yucatan Peninsula & Culexquinquefasciatus & GU289686 \\
\hline 37 & M2614 & 2008 & Mexico & Yucatan Peninsula & Culexquinquefasciatus & GU289687 \\
\hline 38 & M2617 & 2008 & Mexico & Yucatan Peninsula & Culexquinquefasciatus & GU289688 \\
\hline 39 & M2618 & 2008 & Mexico & Yucatan Peninsula & Culexquinquefasciatus & GU289689 \\
\hline 40 & M2630 & 2008 & Mexico & Yucatan Peninsula & Culexquinquefasciatus & GU289690 \\
\hline 41 & M2635 & 2008 & Mexico & Yucatan Peninsula & Culexquinquefasciatus & GU289691 \\
\hline 42 & M2636 & 2008 & Mexico & Yucatan Peninsula & Culexquinquefasciatus & GU289692 \\
\hline 43 & M2637 & 2008 & Mexico & Yucatan Peninsula & Culexquinquefasciatus & GU289693 \\
\hline 44 & M2644 & 2008 & Mexico & Yucatan Peninsula & Culexquinquefasciatus & GU289694 \\
\hline 45 & M2645 & 2008 & Mexico & Yucatan Peninsula & Culexquinquefasciatus & GU289695 \\
\hline 46 & M2648 & 2008 & Mexico & Yucatan Peninsula & Culexquinquefasciatus & GU289696 \\
\hline
\end{tabular}


Table 2 Details of Culexflavivirus (CxFV) strains used in this study (Continued)

\begin{tabular}{lllllll}
\hline 47 & M2650 & 2008 & Mexico & Yucatan Peninsula & Culexquinquefasciatus & GU289697 \\
\hline 48 & M2656 & 2008 & Mexico & Yucatan Peninsula & Culexquinquefasciatus & GU289698 \\
\hline 49 & M2663 & 2008 & Mexico & Yucatan Peninsula & Culexquinquefasciatus & GU289699 \\
\hline 50 & M2665 & 2008 & Mexico & Yucatan Peninsula & Culexquinquefasciatus & GU289700 \\
\hline
\end{tabular}

*Sequence of SDDM06-11 was used in the present study.

nucleotide identity occurred in the NS2a and NS2b genes, where the nucleotide identity between SDDM0611 and genotype 1 strains was $>98 \%$ and $>96 \%$ respectively;. For genotype 2, the identity was $>94 \%$ and $>$ $92 \%$ respectively. however, the amino acid homology was lower than ORF (Table 5).

\section{Phylogenetic analysis}

The estimated ML phylogenetic tree based on the $\mathrm{E}$ gene nucleotide sequence is shown in Figure 3 and is consistent with the trees estimated for the using the NJ and ML methods using MEGA v5.1 (data not shown). The tree shows the CxFV strains are divided into two distinct genotypes. Genotype 1 is divided into two clades, with clade 1 incorporating sequences from Japan and Indonesia and clade 2 containing samples from the United States (Texas and Iowa) [8]. Genotype 2 contains samples from Africa (Uganda), North America (Mexico), Central America (Guatemala) and the West Indies, (Trinidad) [7]. The newly isolated Chinese strain SDDM0611 is placed within the genotype 1 clade; however, it appears to have branched from the remaining isolates at a relatively early time point, suggesting that it belongs to a distinct cluster (Figure 3).

\section{Discussion}

We report here the first isolation of a strain of CxFV in China. This strain is distinct from other isolates collected from around the world, containing signature amino acid mutations and forming a separate cluster in

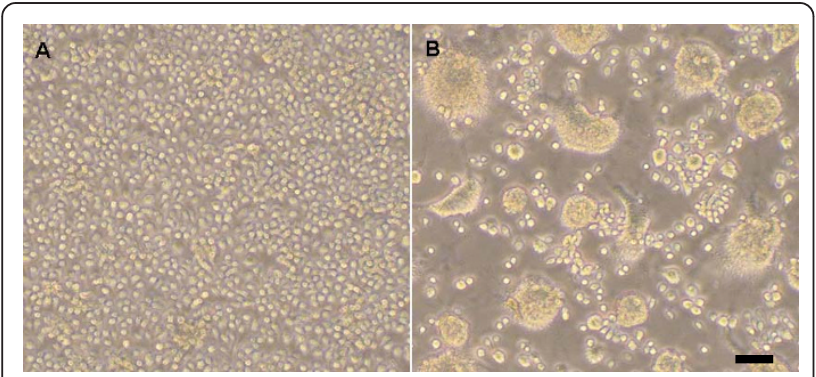

Figure 1 Phase contrast photomicrographs of control and infected C6/36 cells after infection. (A) Control cells. (B) Cells 3 days after infection with SDDM06-11. Note the extensive cell fusion and syncytia formation in the infected cells. Microscope settings Ocular: 10; Lens: 10X. Scale bar, $50 \mu \mathrm{m}$. the estimated phylogenetic tree. This sample appears therefore to represent a new cluster within genotype 1 ; this is also supported by the analysis of the amino acid substitutions in the E protein; many of the mutations were shared by these genotypes, whereas SDDM06-11 contains several unique substitutions.

Investigation of the CPE of this strain also produced distinctive results. Previous studies of CPE produced by $\mathrm{CxFV}$ in C6/36 cells found that some genotype 1 strains could generate marked CPE in cells within 6 to 7 days but no such changes have been observed for genotype 2 strains $[7,9,10]$. For the SDDM06-11 from China the CPE was similar to observations for genotype $1[6,8]$, producing marked CPE but with a more rapid onset of effects (3-4 days) This difference may be a consequence of the detected amino acid changes in the $\mathrm{E}$ gene, but detailed experimental studies are necessary to gain further insight and understanding of the observed differences between the two genotypes.

The genotypes are formed with high bootstrap support and contain clear geographical subdivision, but on a global scale the geographical distribution is less clear with North America (Texas and Iowa), Japan and the Chinese isolate placed in genotype 1 and the Central America (Guatemala and Mexico), Trinidad and Africa strains placed in genotype 2 . Similarly, the evolutionary relationship among the three genotypes is not apparent from the estimated phylogenetic tree. This is primarily because there are relatively few samples available for the analysis and only a partial evolutionary history of the virus can be recreated.

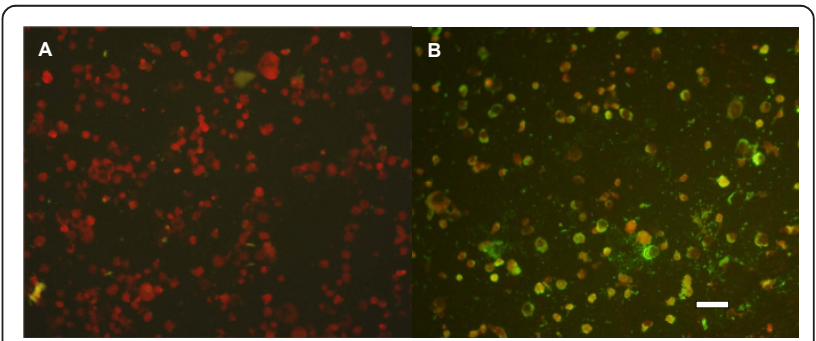

Figure 2 Immunofluorescence assay (IFA) of SDDM06-11 using JEV polyclonal antibody (JEV-GSS). (A) C6/36 cell control

(uninfected) stained by IFA, using JEV polyclonal antibody (JEV-GSS). (B) SDDM06-11 antigen in infected C6/36 cells, as detected by IFA, using JEV polyclonal antibody (JEV-GSS). Scale bar, $50 \mu \mathrm{m}$. 
Table 3 Percentage nucleotide/amino acid identity for the envelope genes among Culexflavivirus isolates from different countries and genotypes

\begin{tabular}{|c|c|c|c|c|c|c|c|c|c|c|}
\hline & & \multicolumn{5}{|c|}{ GENOTYPE 1} & \multicolumn{4}{|c|}{ GENOTYPE 2} \\
\hline & & China & Japan & Indonesia & Texas & lowa & Mexico & Guatemala & Trinidad & Uganda \\
\hline \multirow{5}{*}{ GEN 1} & China & - & 94.6 & 94.8 & 94.5 & 94.4 & 89.4 & 89.7 & 89.6 & 90.2 \\
\hline & Japan & 98.6 & - & 97.5 & 97.4 & 97.4 & 89.1 & 89.3 & 89.3 & 90.2 \\
\hline & Indonesia & 98.4 & 99.3 & - & 97.4 & 97.3 & 89.9 & 90.1 & 90.1 & 90.9 \\
\hline & Texas & 98.6 & 99.5 & 99.3 & - & 99.6 & 89.8 & 89.9 & 89.5 & 90.6 \\
\hline & lowa & 98.6 & 99.5 & 99.3 & 100 & - & 90 & 90.2 & 89.8 & 90.8 \\
\hline \multirow{4}{*}{ GEN 2} & Mexico & 97.9 & 97.4 & 97.2 & 97.4 & 97.4 & - & 99.2 & 98.4 & 97.9 \\
\hline & Guatemala & 97.9 & 97.4 & 97.2 & 97.4 & 97.4 & 100 & - & 98.4 & 97.9 \\
\hline & Trinidad & 97.9 & 97.4 & 97.2 & 97.4 & 97.4 & 100 & 100 & - & 98 \\
\hline & Uganda & 97.9 & 97.4 & 97.2 & 97.4 & 97.4 & 99.5 & 99.5 & 99.5 & - \\
\hline
\end{tabular}

Percentage nucleotide and amino acid homology between and amongst genotypes and countries; nucleotide divergence is displayed in bold type in upper triangle, amino acid homology is displayed in regular type in lower triangle. Alignments were performed using the following CxFV isolates: SDDM06-11 (China), NIID-21-2 (Japan), Surabaya (Indonesia), TX 24518 (Texas), 380 (lowa) belonging to Genotype 1 and CxFV-Mex07 (Mexico), Izabal (Guatemala), TR3116 (Trinidad) and Uganda08 (Uganda) belonging to Genotype 2.

Table 4 Deduced amino acid substitutions for CxFV by genotype in the E gene

\begin{tabular}{|c|c|c|c|c|c|c|c|c|c|c|c|c|}
\hline \multirow[t]{2}{*}{ Genotype $^{1}$} & \multicolumn{12}{|c|}{ Residue $^{2}$} \\
\hline & $E-60$ & $E-64$ & E-117 & $\mathrm{E}-125$ & E-185 & E-236 & $\mathrm{E}-326$ & $E-340$ & E-381 & E-407 & E-414 & E-423 \\
\hline G1 & V (Val) & F (Phe) & G (Gly) & I (lle) & $\mathrm{H}$ (His) & V ( Val) & R (Arg) & V (Val) & $\mathrm{H}$ (His) & F (Phe) & F (Phe) & V (Val) \\
\hline $\mathrm{G} 2$ & $A^{3}$ (Ala) & Y (Tyr) & E (Glu) & I (lle) & $\mathrm{Y}(\mathrm{Tyr})$ & I (Ile) & K (Lys) & I (lle) & $\mathrm{Y}(\mathrm{Tyr})$ & L (Leu) & F (Phe) & I (lle) \\
\hline SDDM06-11 & V ( Val) & F (Phe) & G (Gly) & V (Val) & $\mathrm{H}$ (His) & V (Val) & $\mathrm{R}$ (Arg) & I (lle) & $\mathrm{H}$ (His) & L (Leu) & V (Val) & I (lle) \\
\hline
\end{tabular}

1G1: Japan and Indonesia, USA:TX/lowa; G2: Uganda, Guatemala, Mexico, West Indies;

${ }^{2}$ Amino acid positions are determined from the sequence alignment of the completely $\mathrm{E}$ sequenced isolates of $\mathrm{CxFV}$.

${ }^{3}$ Boldface indicates deduced amino acid specific to that genotype.

All CxFV samples to date have been isolated from members of the Culex genus, with the majority of samples collected from Culexpipiens and Culexquinquefasciatus. Genotype 2 primarily consists of Culexquinquefasciatus isolates, whereas genotype 1 is primarily Culexpipiens and contains smaller numbers of other Culex species [6-11]. The SDDM06-11 belonging to genotype 1 is also isolated from Culexpipiens.

An earlier theoretical study of species diversification derived an estimate for the relationship between the distribution of internal nodes in a tree predicted from a limited sample set and the number of different viruses from which the sample set were selected [21]. When applied to the flaviviruses genus, it was estimated that are several thousand unsampled mosquito borne flaviviruses [21]. Thus further collection and evaluation of CxFV samples is necessary to gain further insight into the evolutionary history of the virus and the host range. It is likely that this will reveal greater complexity and structure (i.e. additional genotypes and new strains) in the CxFV phylogeny and host population.

Table 5 Nucleotide and amino acid of ORF sequence comparison (\% sequence identity) of strain SDDM06-11 with selected CxFV isolates

\begin{tabular}{cccccccccccc}
\hline SDDM06-11 & $\mathbf{C}$ & PrM & $\mathbf{E}$ & NS1 & NS2a & NS2b & NS3 & NS4a & NS4b & NS5 & Total \\
\cline { 2 - 12 } & $417(139)^{\mathrm{a}}$ & $429(143)$ & $1281(427)$ & $1107(369)$ & $690(230)$ & $381(127)$ & $1779(593)$ & $567(189)$ & $771(257)$ & $2667(889)$ & $10089(3363)$ \\
\hline Tokyo & $94.5(95.0)^{b}$ & $95.3(98.6)$ & $94.6(98.6)$ & $94.7(99.2)$ & $98.3(97.8)$ & $96.9(94.5)$ & $94.3(97.5)$ & $94.0(98.4)$ & $94.0(97.3)$ & $94.7(98.7)$ & $94.9(98.0)$ \\
\hline NIID-21-2 & $94.7(95.0)$ & $95.6(99.3)$ & $94.6(98.6)$ & $94.8(99.2)$ & $98.3(97.8)$ & $96.9(94.5)$ & $94.5(97.6)$ & $94.0(98.4)$ & $94.0(97.3)$ & $95.0(99.1)$ & $95.0(98.2)$ \\
\hline lowa07 & $95.0(94.2)$ & $95.6(99.3)$ & $95.0(98.8)$ & $94.8(99.2)$ & $98.6(98.3)$ & $96.6(94.5)$ & $94.5(97.5)$ & $94.0(98.4)$ & $94.2(97.3)$ & $95.1(99.3)$ & $95.1(98.2)$ \\
\hline HOU24518 & $95.0(95.0)$ & $94.6(98.6)$ & $94.5(98.6)$ & $94.4(99.2)$ & $98.3(98.3)$ & $96.1(95.3)$ & $94.2(97.5)$ & $94.4(99.5)$ & $94.4(96.9)$ & $94.6(98.7)$ & $94.8(98.1)$ \\
\hline CxFV-Mex07 & $91.8(89.9)$ & $92.8(98.6)$ & $89.4(97.9)$ & $91.3(98.1)$ & $94.8(93.9)$ & $92.4(93.7)$ & $88.8(96.5)$ & $87.5(96.3)$ & $88.8(95.7)$ & $89.8(97.5)$ & $90.2(96.6)$ \\
\hline UGANDA08 & $92.3(91.4)$ & $93.0(97.9)$ & $90.2(97.9)$ & $92.0(98.1)$ & $94.8(94.3)$ & $92.9(94.5)$ & $89.3(96.5)$ & $88.4(96.3)$ & $87.7(94.2)$ & $89.8(97.2)$ & $90.5(96.5)$ \\
\hline
\end{tabular}

a Nucleotide number were ahead and amino acid number shown in brackets.

${ }^{b}$ Percent nucleotide sequence homology were ahead and amino acid sequence homology shown in brackets. 


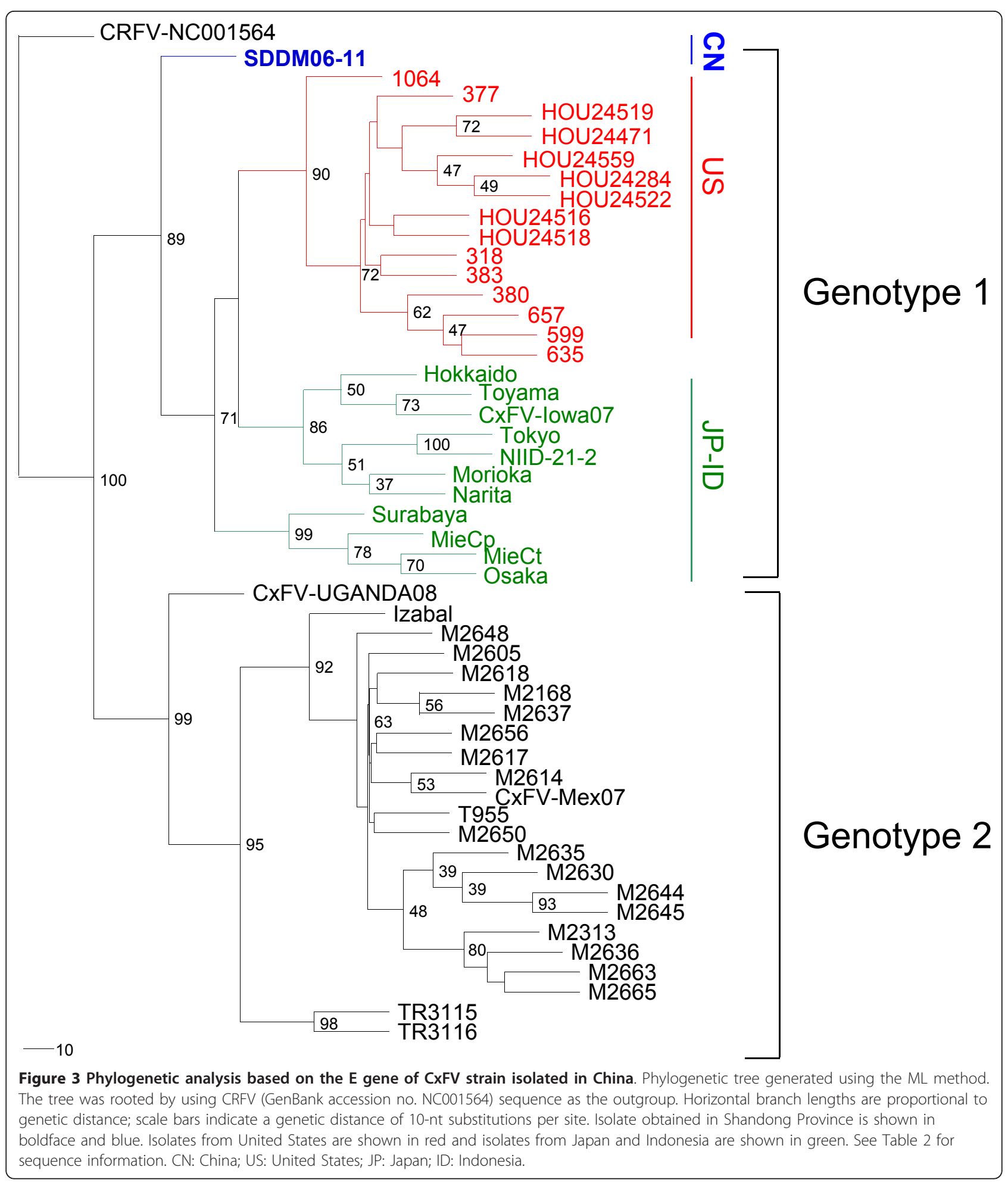

\section{Conclusions}

We have isolated and identified the CxFV strain in mainland China. Phylogenetic analysis places the isolate within genotype 1 , but indicates it is quite distinct from other strains within this clade. This is further supported by analysis of the $\mathrm{E}$ gene and ORF sequence which shows the isolate has discrete difference in terms of nucleotide composition and amino acid mutations. Our 
findings suggest that CxFV may be widespread in Culex mosquito populations and could have a relevant role in the evolution of flaviviruses.

\section{Abbreviations}

CPE: Cytopathic Effect; CxFV: CulexFlavivirus; IFA: immunofluorescence assay

\section{Acknowledgements}

We thank the staff from Shandong Province Centers for Disease Control and Prevention and the two local Centers for Disease Control and Prevention (Heze city and Dongmingcounty) for assistance with the mosquito collection.

This work was supported by grants from the Ministry of Science and Technology of People's Republic of China (No. 2003BA712A08-01 and No. 2008ZX10004-010), the National Natural Science Foundation of China (31070145), Development Grant of State Key Laboratory for Infectious Disease Prevention and Control (2008SKLID105).

\section{Author details}

State Key Laboratory for Infectious Disease Prevention and Control, Department of Viral Encephalitis, Institute for Viral Disease Control and Prevention, Chinese Center for Disease Control and Prevention, Beijing 102206, People's Republic of China. ${ }^{2}$ Institute for Immunization Program, Shandong province Center for Disease Control and Prevention, Jinan, Shandong 250014, People's Republic of China. ${ }^{3}$ Bioinformatics Group, State Key Laboratory for Virology, Wuhan Institute of Virology, Chinese Academy of Sciences, Wuhan, Hubei 430071, People's Republic of China.

\section{Authors' contributions}

Wang Huanyu', Wang Haiyan², Fu Shihong ', Liu Guifang ${ }^{2}$, Liu Hong', Gao Xiaoyan', Song Lizhi', Simon Rayner', Xu Aiqiang', Liang Guodong ${ }^{3 *}$

WHYu carried out mosquito collection, virus isolation, nucleic acid detection and sequencing, participated in the sequence alignment, phylogenetic analysis and drafted the manuscript. WHYan, LGF, SLZ and XAQ participated in the mosquito collection. FSH, LH and GXY participated in the virus isolation, nucleic acid detection and phylogenetic analysis. SR participated in discussions and data analysis. LGD conceived the study and participated in its design and coordination. All authors read and approved the final manuscript.

\section{Authors' information}

Dr. Wang Huanyu, Ph.D., is an associate professor at the State Key Laboratory for Infectious Disease Prevention and Control, the Institute for Viral Disease Control and Prevention, Chinese Center for Disease Control and Prevention. His current research focuses on arbovirus and viral encephalitis, especially in Flavivirus.

\section{Competing interests}

The authors declare that they have no competing interests.

Received: 19 October 2011 Accepted: 27 March 2012

Published: 27 March 2012

\section{References}

1. Fields Virology. Edited by: Fields BN, Knipe DM, Howley PM. Lippincott Williams, 5 2007:1154-1228.

2. Gaunt MW, Sall AA, de Lamballerie X, Falconar AK, Dzhivanian TI, Gould EA: Phylogenetic relationships of flaviviruses correlate with their epidemiology, disease association and biogeography. J Gen Virol 2001, 82(Pt 8):1867-1876.

3. Kuno G, Chang GJ, Tsuchiya KR, Karabatsos N, Cropp CB: Phylogeny of the genus Flavivirus. J Virol 1998, 72(1):73-83.

4. Stollar V, Thomas VL: An agent in the Aedesaegypti cell line (Peleg) which causes fusion of Aedesalbopictus cells. Virology 1975, 64(2):367-377.

5. Crabtree MB, Sang RC, Stollar V, Dunster LM, Miller BR: Genetic and phenotypic characterization of the newly described insect flavivirus, Kamiti River virus. Arch Virol 2003, 148(6):1095-1118.
6. Hoshino K, Isawa H, Tsuda Y, Yano K, Sasaki T, Yuda M, Takasaki T, Kobayashi M, Sawabe K: Genetic characterization of a new insect flavivirus isolated from Culexpipiens mosquito in Japan. Virology 2007, 359(2):405-414.

7. Blitvich BJ, Lin M, Dorman KS, Soto V, Hovav E, Tucker BJ, Staley M, Platt KB, Bartholomay LC: Genomic sequence and phylogenetic analysis of Culexflavivirus, an insect-specific flavivirus, isolated from Culexpipiens (Diptera: Culicidae) in lowa. J Med Entomol 2009, 46(4):934-941.

8. Kim DY, Guzman H, Bueno R Jr, Dennett JA, Auguste AJ, Carrington CV, Popov VL, Weaver SC, Beasley DW, Tesh RB: Characterization of CulexFlavivirus (Flaviviridae) strains isolated from mosquitoes in the United States and Trinidad. Virology 2009, 386(1):154-159.

9. Morales-Betoulle ME, Monzon Pineda ML, Sosa SM, Panella N, Lopez MR, Cordon-Rosales C, Komar N, Powers A, Johnson BW: Culexflavivirus isolates from mosquitoes in Guatemala. J Med Entomol 2008, 45(6):1187-1190.

10. Farfan-Ale JA, Lorono-Pino MA, Garcia-Rejon JE, Hovav E, Powers AM, Lin M, Dorman KS, Platt KB, Bartholomay LC, Soto V, et al: Detection of RNA from a novel West Nile-like virus and high prevalence of an insect-specific flavivirus in mosquitoes in the Yucatan Peninsula of Mexico. Am J Trop Med Hyg 2009, 80(1):85-95.

11. Saiyasombat R, Dorman KS, Garcia-Rejon JE, Lorono-Pino MA, Farfan-Ale JA, Blitvich BJ: Isolation and sequence analysis of Culexflavivirus from Culex interrogator and Culexquinquefasciatus in the Yucatan Peninsula of Mexico. Arch Virol 2010, 155(6):983-986.

12. Cook S, Moureau G, Harbach RE, Mukwaya L, Goodger K, Ssenfuka F, Gould E, Holmes EC, de Lamballerie X: Isolation of a novel species of flavivirus and a new strain of Culexflavivirus (Flaviviridae) from a natural mosquito population in Uganda. J Gen Virol 2009, 90(Pt 11):2669-2678.

13. Wang $H$, Li Y, Liang $X$, Liang G: Japanese encephalitis in mainland china. Jpn J Infect Dis 2009, 62(5):331-336.

14. Gao X, Nasci R, Liang G: The neglected arboviral infections in mainland China. PLoS Negl Trop Dis 2010, 4(4):e624.

15. Wang ZS AS, Wang Y, Han R, Guo JQ: A new virus of flavivirus: Chaoyang virus isolated in Liaoning province. Chin J Public Health 2009, 25(7):3.

16. LU BL: Identification of mosquitoes. In Classification and Identification of Important Medical Insects of China. Edited by: Lu, BL, Wu, YH. Zhengzhou: Henan Science and Technology Publishing House; 2003:12-105, in Chinese.

17. Li WJ, Wang JL, Li MH, Fu SH, Wang HY, Wang ZY, Jiang SY, Wang XW, Guo P, Zhao SC, et al: Mosquitoes and mosquito-borne arboviruses in Qinghai-Tibet Plateau-Focus on the Qinghai area, China. Am J Trop Med Hyg 2010, 82(4):705-711.

18. Liang GD, He Y, Chen BQ, Zhao ZJ, Huang YJ, Chen L: Preparation of arbovirus group-specific PcAb and their use to identify newly isolated virus strains. Zhonghua Shi Yan He Lin Chuang Bing Du Xue Za Zhi 1993, 7(4):374-376, in Chinese.

19. Thompson JD, Gibson TJ, Higgins DG: Multiple sequence alignment using ClustalW and ClustalX. Curr Protoc Bioinformatics 2002, Chapter 2, Unit 2 3.

20. Guindon S, Delsuc F, Dufayard JF, Gascuel O: Estimating maximum likelihood phylogenies with PhyML. Methods Mol Biol 2009, 537:113-137.

21. Pybus OG, Rambaut $A$, Holmes EC, Harvey PH: New inferences from tree shape: numbers of missing taxa and population growth rates. Syst Biol 2002, 51(6):881-888.

doi:10.1186/1743-422X-9-73

Cite this article as: Huanyu et al:: Isolation and identification of a distinct strain of Culex Flavivirus from mosquitoes collected in Mainland China. Virology Journal 2012 9:73. 